\title{
Cylinder Test to Assess Sensory-motor Function in a Mouse Model of Parkinson's Disease
}

Luiz Alexandre Viana Magno1, *, Mélcar Collodetti ${ }^{1}$, Helia Tenza-Ferrer ${ }^{1}$ and Marco Aurélio Romano-Silva ${ }^{1,2, *}$

\begin{abstract}
${ }^{1}$ Centro de Tecnologia em Medicina Molecular, Faculdade de Medicina, Universidade Federal de Minas Gerais (UFMG), Belo Horizonte, CEP 30130-100, Brazil; ²Departamento de Saúde Mental, Faculdade de Medicina, Universidade Federal de Minas Gerais (UFMG), Belo Horizonte, CEP 30130-100, Brazil *For correspondence: lavmagno@ufmg.br; romano-silva@ufmg.br
\end{abstract}

[Abstract] Parkinson's disease is a progressive neurodegenerative movement disorder that happens due to the loss of dopaminergic neurons in the substantia nigra. The deficiency of dopamine in the basal nuclei drives cardinal motor symptoms such as bradykinesia and hypokinesia. The current protocol describes the cylinder test, which is a relatively simple behavioral assessment that evaluates the motor deficits upon unilateral degeneration of the nigrostriatal pathway in experimental models of Parkinson's disease. Since dopamine-depleted mice exhibit the preferential use of the forelimb ipsilateral to the lesion, here researchers perform the cylinder test to investigate the therapeutic effects of antiparkinsonian treatments on the performance of the contralateral (injured) limb.

Keywords: Cylinder test, Parkinson's disease, Stroke, Motor system, Behavior

[Background] The cylinder test measures the rodents spontaneous forelimb use, which can be used to evaluate the sensory-motor function in a number of injury models that cause forelimb use asymmetry (Schallert et al., 2000). In this test, the mouse is placed in a glass cylinder and the number of times it rears up and touches the cylinder wall is measured. The wall touches are subsequently scored for the left, right, or both paws by an observer in slow motion recorded videos. The results are expressed as the percentage of each paw usage relative to the total number of touches.

The cylinder test is a common behavioral assessment used to evaluate the motor impairments in experimental models of Parkinson's disease. Our laboratory and others have previously shown that mice with unilateral injection of 6-OHDA in the striatum, substantia nigra pars compacta or medial forebrain bundle exhibit a remarkable forelimb asymmetry due to lesion of the nigrostriatal pathway (lancu et al., 2005; Kriks et al., 2011; Subramaniam et al., 2011; Boix et al., 2015; Chu et al., 2017; Magno et al., 2019). While a healthy mouse uses the right and the left paw indifferently, dopamine-lesioned mice show preferential use of the paw ipsilateral to the lesion (Figure 1).

The cylinder test can be used to evaluate the preclinical screening of antiparkinsonian therapeutic interventions based on the functional recovery of the contralateral paw (Kriks et al., 2011; Francardo et al., 2014; Fischer et al., 2017; Magno et al., 2019). A number of other unilateral experimental models of neurological disorder including spinal cord injury and cerebral ischemia have been shown to be sensitive to the degree of forelimb asymmetry measured in the cylinder test (Schallert et al., 2000; Starkey et al., 2005; Venna et al., 2014; Wang et al., 2015; Warren et al., 2018; Carballo-Carbajal et al., 2019). 
The cylinder test has been found to be easy to perform and sensitive to detect motor impairments that other behavioral tests fail to detect. On the other hand, analysis of the videotape records are timeconsuming, and the test cannot be repeated frequently as mice will lose interest in exploring the vertical surface of the cylinder (Lundblad et al., 2002).

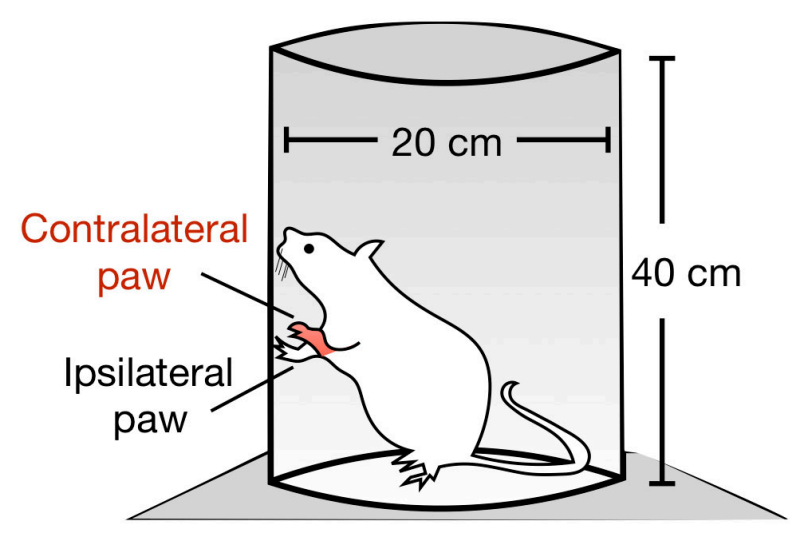

Figure 1. Parkinson's disease mouse model in the Cylinder Test. The contralateral paw is located in the opposite side of the 6-OHDA unilateral injection. For example, the left paw is considered contralateral when 6-OHDA is injected in the right brain hemisphere. Unilateral lesion of the striatum with 6-OHDA causes preferential use of the forelimb ipsilateral paw (the paw in the same side of the lesion).

\section{Materials and Reagents}

1. Black curtain

2. Mice

3. Ethanol $70 \%$ (Ciclo Pharma, supplied by Prime Cirúrgica, catalog number: 67 )

4. Soap (Ciclo Pharma, supplied by Prime Cirúrgica, catalog number: 61)

\section{Equipment}

1. Glass cylinder $20 \mathrm{~cm}$ in diameter and approximately $40 \mathrm{~cm}$ in height In our lab, we use a $2 \mathrm{~L}$ graduated beaker made of borosilicate glass (Global Glass, catalog number: GTBFB-2000).

2. Three digital cameras capable to record videos at $15-25$ frames per second, $1,280 \times 720$ pixels of resolution, and fulfilled with built-in infra-red (IR) illumination and onboard storage (TP-link, catalog number: NC250)

3. Timer (Fisher Scientific, Traceable Nano, catalog number: 14-649-83)

4. Any computer or TV capable of running the recorded video files 


\section{Software}

1. Any media player with video support and capability to change the playback speed. In our lab, we use the VLC Media Player v3.0.6 (VideoLAN, http://www.videolan.org/vlc/)

2. GraphPad Prism (GraphPad Software, http://www.graphpad.com)

\section{Procedure}

A. Experimental preparation

1. It is best to conduct the cylinder test during the mice's active phase (dark cycle).

Note: It is convenient to maintain all tested mice on a reverse light/dark cycle.

2. Mark the mice's tails using a waterproof marking pen on the day before of the experiment. This will allow quick identification of mice in the cage and avoid a high level of stress immediately before testing.

3. Run all behavioral evaluations at the same time of the day.

4. Place the cylinder on the center of the table or lab bench. Use a black curtain to cover all sides of the cylinder to minimize visual distraction to the mice.

Note: Draw a line on the surface where the cylinder sits to ensure the cylinder can be returned to the same position.

5. Position the top-view camera above the cylinder. The view should be close enough to acquire the entire diameter of the cylinder (Figure 2). The other two cameras must cover the side of the cylinder (side-view cameras) (Figure 3B).

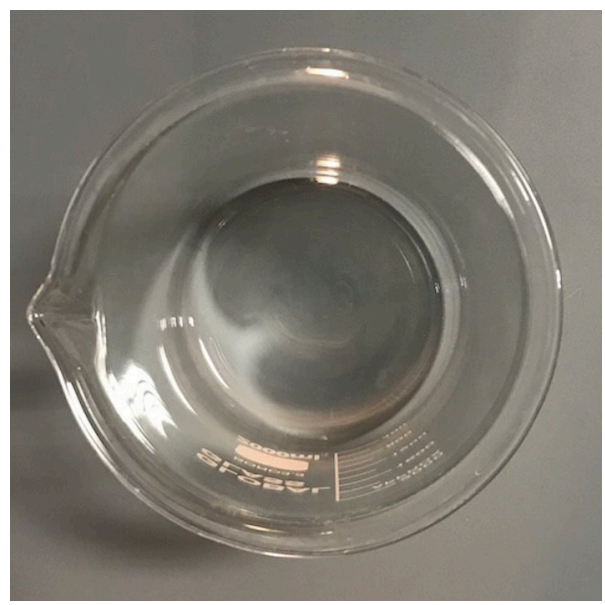

Figure 1. View from the top camera

6. Perform the test under low light conditions. The dark environment will stimulate the exploratory instinct of mice. In our animal facility, we use a ceiling white light as the main source of illumination. This light source is controlled by a dimmer switch set to deliver 40 lux above the cylinder. 
Please cite this article as: Magno et. al., (2019). Cylinder Test to Assess Sensory-motor Function in a Mouse Model of Parkinson's Disease,Bio-protocol 9

Note: Ensure the lighting conditions are enough to clearly see the forelimb use around the cylinder in the video recordings.

7. Prepare test cards to identify each animal during the video recording. However, do not indicate the experimental groups to ensure blind scores.

8. The human experimenter conducting the behavioral assay should not be visible to the assayed mouse.

Note: Leave the room while the mice are performing the experiment.

9. Acclimate the mice into the testing room for at least $30 \mathrm{~min}$ before testing. Provide the same light conditions used for the test. Allow mice to rest undisturbed.

B. Behavioral assay

1. Start video recording.

2. Label a test card with animal identification and record it for $3 \mathrm{~s}$.

3. Place the mouse into the middle of the glass cylinder and start the timer (Figure 3).

A

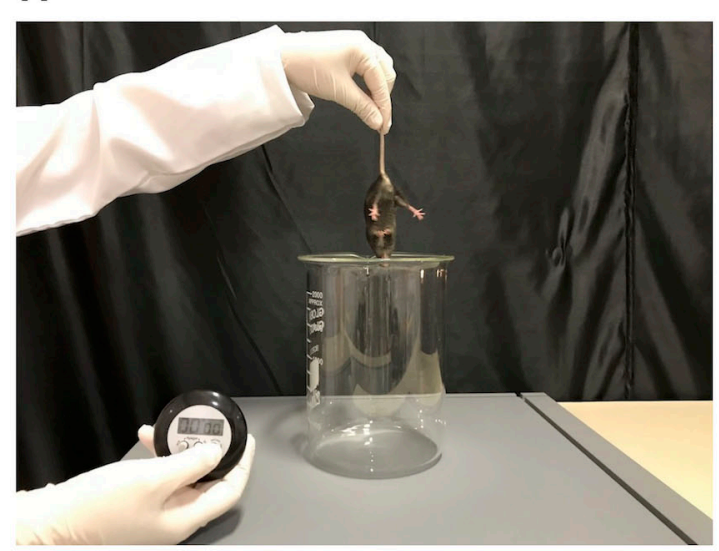

B

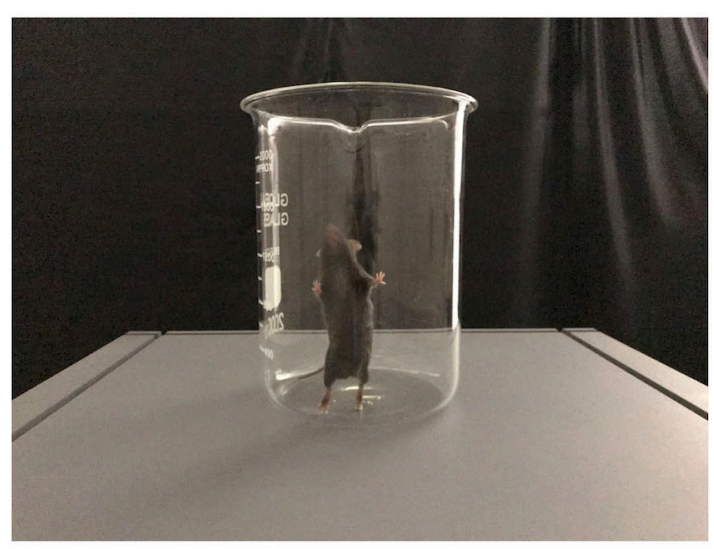

Figure 2. The cylinder test. A. Mice are placed in the center of the cylinder. B. View from the side-view camera showing a simultaneous forelimb wall contact.

4. Record the mice for $10 \mathrm{~min}$. At the end of the test, return the mouse immediately to its homecage. Table 1 shows representative data from one mouse.

Table 1. Representative number of paw touches of a mouse in a 10-min test

\begin{tabular}{ll}
\hline Forelimb use & Number of touches \\
\hline Left paw & 16 \\
Right paw & 14 \\
Both paws & 27 \\
\hline
\end{tabular}

5. Clean the cylinder with soap and water.

6. Spray the cylinder with $70 \%$ ethanol to remove any odorant trace. 
7. Allow the glass cylinder to dry before the next test.

8. Repeat Steps B2-B7 for all tested mice.

9. Return all mice to the housing room.

\section{Data analysis}

Note: The video recorded sessions should be scored by an experimenter unaware of the treatment of the mouse.

1. Open Video 1 in a slow-motion mode.

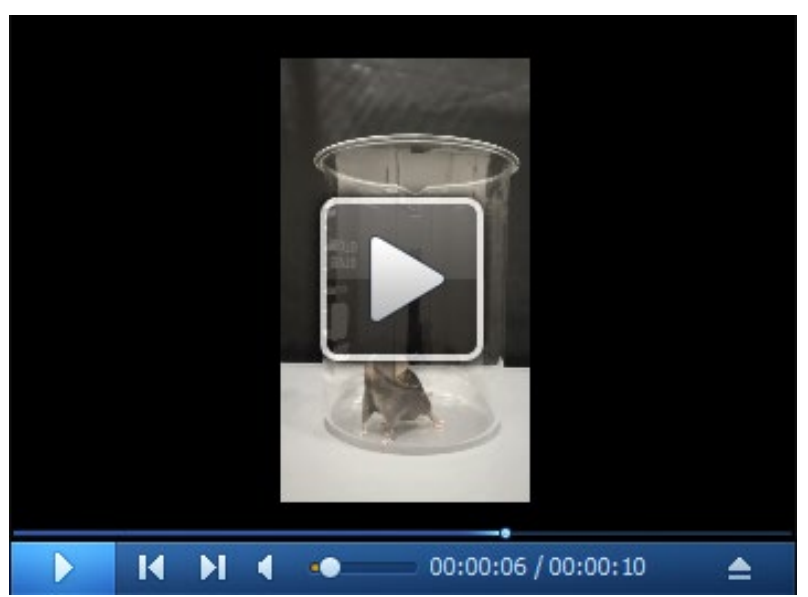

Video 1. Video from the side-view camera. (All animal procedures were carried out in accordance with the National Institute of Health guidelines and approved by the Institutional Animal Ethics Committee of the Universidade Federal de Minas Gerais (protocol 59/2014).)

2. Score each time the mouse rears up (a vertical movement in which the mouse is standing only on its hindlimbs) and places the paw on the wall of the cylinder. Wall touches are counted for the right, left or both paws (in simultaneous double contacts). Use the video recorded from the side-view cameras to score the behavior when the mice turn away from the top-view camera.

3. Exclude simultaneous paw touches from the analysis. Express data as a percentage of impaired forelimb relative to the total contacts [(contralateral touches)/(ipsilateral touches + contralateral touches) $\times 100]$ (Figure 4).

4. Use a parametric analysis such as the unpaired Student's $t$-test for two-sample comparisons. We perform our statistical analysis and produce graphs with the GraphPad Prism version 6 (GraphPad Software). 


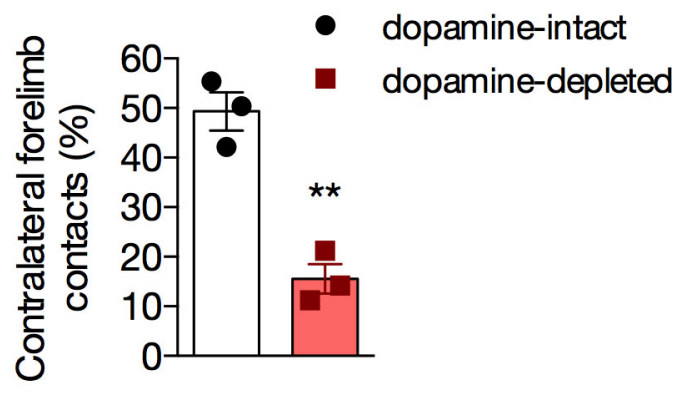

Figure 3. Unilateral dopamine-depleted mice exhibit forelimb use asymmetry detected in Cylinder Test ${ }^{* \star} P<0.05$, Student's $t$-test, $\mathrm{n}=3$ /group)

\section{$\underline{\text { Notes }}$}

1. The size of the glass cylinder may vary according to the size of the mouse strain. Bigger or more active strains can attempt to escape by jumping onto the cylinder's rim. In this case, taller cylinders should be used. The cylinder we used in this protocol is appropriate for the C57BL/6 mice.

2. Some researchers use mirrors placed next to the cylinder to ensure views of the forelimbs in the video recordings.

3. Do not habituate the mice to the cylinder prior to testing. This can cause mice to show little or no tendency to explore.

4. The trial duration varies among the protocols. A 5 to 10 -min trial is enough to obtain a sufficient number of movements for reliable measurement. Most mice are less active after this period.

5. It is best to perform this test in a soundproof room as loud noises can distract the mouse. If this is not possible, add a sign showing "Experiment in Progress" on the door and nearby area to eliminate any conversation.

6. Most studies typically use between 8 and 10 mice per group.

7. The procedure for the 6-OHDA-induced lesion of the nigrostriatal pathway was described in the previously published paper (Magno et al., 2019)

\section{Acknowledgments}

This protocol was adapted from Magno et al. (2019). This study was financed in part by the Coordenação de Aperfeiçoamento de Pessoal de Nível Superior - Brasil (CAPES) - Finance Code 001 to L.A.V.M and H.T.-F., Fundação de Amparo à Pesquisa do Estado de Minas Gerais (FAPEMIG APQ 00476-14) to M.A.R.-S., and Universal CNPq 457639/2014-8 to L.A.V.M.

\section{Competing interests}

The authors declare no competing financial interests. 
Please cite this article as: Magno et. al., (2019). Cylinder Test to Assess Sensory-motor Function in a Mouse Model of Parkinson's Disease,Bio-protocol 9 (16): e3337. DOI: 10.21769/BioProtoc.3337.

\section{Ethics}

All animal procedures were carried out in accordance with the National Institute of Health guidelines and approved by the Institutional Animal Ethics Committee of the Universidade Federal de Minas Gerais (protocol 59/2014).

\section{$\underline{\text { References }}$}

1. Boix, J., Padel, T. and Paul, G. (2015). A partial lesion model of Parkinson's disease in mice-characterization of a 6-OHDA-induced medial forebrain bundle lesion. Behav Brain Res 284: 196-206.

2. Carballo-Carbajal, I., Laguna, A., Romero-Gimenez, J., Cuadros, T., Bove, J., Martinez-Vicente, M., Parent, A., Gonzalez-Sepulveda, M., Penuelas, N., Torra, A., Rodriguez-Galvan, B., Ballabio, A., Hasegawa, T., Bortolozzi, A., Gelpi, E. and Vila, M. (2019). Brain tyrosinase overexpression implicates age-dependent neuromelanin production in Parkinson's disease pathogenesis. Nat Commun 10(1): 973.

3. Chu, H. Y., Mclver, E. L., Kovaleski, R. F., Atherton, J. F. and Bevan, M. D. (2017). Loss of hyperdirect Pathway cortico-subthalamic inputs following degeneration of midbrain dopamine neurons. Neuron 95(6): 1306-1318 e1305.

4. Fischer, D. L., Manfredsson, F. P., Kemp, C. J., Cole-Strauss, A., Lipton, J. W., Duffy, M. F., Polinski, N. K., Steece-Collier, K., Collier, T. J., Gombash, S. E., Buhlinger, D. J. and Sortwell, C. E. (2017). Subthalamic nucleus deep brain stimulation does not modify the functional deficits or axonopathy induced by nigrostriatal a-synuclein overexpression. Sci Rep 7(1): 16356.

5. Francardo, V., Bez, F., Wieloch, T., Nissbrandt, H., Ruscher, K. and Cenci, M. A. (2014). Pharmacological stimulation of sigma-1 receptors has neurorestorative effects in experimental parkinsonism. Brain 137(Pt 7): 1998-2014.

6. Iancu, R., Mohapel, P., Brundin, P. and Paul, G. (2005). Behavioral characterization of a unilateral 6-OHDA-lesion model of Parkinson's disease in mice. Behav Brain Res 162(1): 1-10.

7. Kriks, S., Shim, J. W., Piao, J., Ganat, Y. M., Wakeman, D. R., Xie, Z., Carrillo-Reid, L., Auyeung, G., Antonacci, C., Buch, A., Yang, L., Beal, M. F., Surmeier, D. J., Kordower, J. H., Tabar, V. and Studer, L. (2011). Dopamine neurons derived from human ES cells efficiently engraft in animal models of Parkinson's disease. Nature 480(7378): 547-551.

8. Lundblad, M., Andersson, M., Winkler, C., Kirik, D., Wierup, N. and Cenci, M. A. (2002). Pharmacological validation of behavioural measures of akinesia and dyskinesia in a rat model of Parkinson's disease. Eur J Neurosci 15(1): 120-132.

9. Magno, L. A. V., Tenza-Ferrer, H., Collodetti, M., Aguiar, M. F. G., Rodrigues, A. P. C., da Silva, R. S., Silva, J. D. P., Nicolau, N. F., Rosa, D. V. F., Birbrair, A., Miranda, D. M. and RomanoSilva, M. A. (2019). Optogenetic stimulation of the M2 cortex reverts motor dysfunction in a mouse model of Parkinson's disease. J Neurosci 39(17): 3234-3248. 
10. Schallert, T., Fleming, S. M., Leasure, J. L., Tillerson, J. L. and Bland, S. T. (2000). CNS plasticity and assessment of forelimb sensorimotor outcome in unilateral rat models of stroke, cortical ablation, parkinsonism and spinal cord injury. Neuropharmacology 39(5): 777-787.

11. Starkey, M. L., Barritt, A. W., Yip, P. K., Davies, M., Hamers, F. P., McMahon, S. B. and Bradbury, E. J. (2005). Assessing behavioural function following a pyramidotomy lesion of the corticospinal tract in adult mice. Exp Neurol 195(2): 524-539.

12. Subramaniam, S., Napolitano, F., Mealer, R. G., Kim, S., Errico, F., Barrow, R., Shahani, N., Tyagi, R., Snyder, S. H. and Usiello, A. (2011). Rhes, a striatal-enriched small G protein, mediates mTOR signaling and L-DOPA-induced dyskinesia. Nat Neurosci 15(2): 191-193.

13. Venna, V. R., Xu, Y., Doran, S. J., Patrizz, A. and McCullough, L. D. (2014). Social interaction plays a critical role in neurogenesis and recovery after stroke. Transl Psychiatry 4: e351.

14. Wang, J., Xia, J., Zhang, F., Shi, Y., Wu, Y., Pu, H., Liou, A. K., Leak, R. K., Yu, X., Chen, L. and Chen, J. (2015). Galectin-1-secreting neural stem cells elicit long-term neuroprotection against ischemic brain injury. Sci Rep 5: 9621.

15. Warren, P. M., Steiger, S. C., Dick, T. E., MacFarlane, P. M., Alilain, W. J. and Silver, J. (2018). Rapid and robust restoration of breathing long after spinal cord injury. Nat Commun 9(1): 4843. 